\title{
Problèmes d'extraction \\ et d'analyse de certains composés aromatiques des produits laitiers \\ I. - TECHNIQUES DE CONCENTRATION ET D'EXTRACTION
}

\author{
par \\ J. L. LACRAMPE, M. DHERBOMEZ, \\ Université de Poitiers, Laboratoire de Biologie et Biochimie \\ I.U.T. de La Rochelle, B.P. 536 \\ et F. WEBER \\ Ecole de Laiterie de Nancy (I.N.P.L.)
}

\section{I. - INTRODUCTION}

Certains composés présents à de très faibles concentrations dans les produits alimentaires sont à l'origine de leur goût et de leur odeur. Grâce aux techniques modernes d'analyse, on n'est plus amené, comme par le passé, à traiter des quantités importantes de produits pour obtenir une quantité de "concentré » aromatique suffisante. Il est actuellement possible d'opérer sur des volumes réduits.

En ce qui concerne les produits laitiers, de nombreuses études ont été entreprises depuis une dizaine d'années pour détecter, identifier et doser les composés responsables de l'arôme. Les méthodes utilisées pour obtenir ces résultats sont des adaptations de techniques de laboratoire classiques. Il est évidemment essentiel de pouvoir caractériser et identifier ces corps non seulement du point de vue chimique et physique mais également organoleptique ; ce qui implique la possibilité de fractionner les différents éléments de l'arôme jusqu'à l'obtention de composés purs.

Du point de vue de la fabrication industrielle, ces résultats étant obtenus, on pourra suivre les qualités organoleptiques d'un produit donné et - dans la mesure où la voie de la biosynthèse des composés aromatiques est connue - orienter efficacement le processus d'élaboration du produit. 
Les techniques d'analyse différeront suivant que les produits sont ou non riches en eau. En effet, s'il est exact que la phase grasse participe à l'arôme du produit, il ne faut pas négliger la fraction aqueuse qui joue aussi un rôle important comme l'ont montré certaines études. I] convient de remarquer avec Weurman [102] que toutes les méthodes de concentration (distillation, adsorption, extraction, congélation) utilisent les lois de partage entre deux phases ; la valeur du coefficient de partage dépend de la méthode ainsi que de la nature du composé utilisé. De ce fait, la proportion des constituants de l'extrait obtenu est différente de celle qui préexistait dans le produit original.

Les produits alimentaires sont des milieux complexes et une analyse donnée ne peut conduire qu'à une solution partielle du problème.

La présente étude rassemble les méthodes utilisées pour isoler les différents composants de l'arôme en insistant sur les corps les plus volatils contenus dans les fractions de tête de distillation tels que le diacétyle et l'acétylméthylcarbinol.

Nous présenterons successivement les techniques de concentration et d'extraction de l'arôme puis les méthodes de dosage de certains composés aromatiques importants.

\section{II. - TECHNIQUES ET MATERIELS DE RECUPERATION DES FRACTIONS AROMATIQUES}

\section{A. - Produits à forte teneur en eau}

L'étude peut porter soit sur les substances aromatiques se trouvant dans l'atmosphère avoisinant immédiatement le produit, soit sur les substances contenues dans le produit lui-même, soit enfin sur les deux catégories de composés.

Les techniques dites de " head-space " qui conduisent à l'analyse des vapeurs surmontant le produit sont intéressantes mais incomplètes pour caractériser l'arôme global. Ces méthodes doivent être complétées par des extractions et des concentrations.

\section{LES DIFFÉRENTES MÉTHODES}

On utilise en général des techniques telles que distillation, concentration, extraction, adsorption de façon à obtenir un degré aromatique suffisant sans avoir de pertes et sans introduire de contaminants.

La distillation et l'extraction par les solvants ont été les premières méthodes de concentration mises en œuvre. Parmi les méthodes physiques, l'entraînement des substances volatiles par un courant gazeux 
a été utilisé également avec récupération de l'éluat sur du charbon actif ou dans des pièges réfrigérés.

1) Technique des « HeAD-SPACES »

Cette technique consiste à prélever quelques $\mathrm{ml}$ de gaz au-dessus du produit à l'aide d'une seringue à gaz et à les injecter dans un chromatographe en phase gazeuse. Weurman [100] et Mackay et al. [65] ont utilisé ce procédé. Kepner et al. [49] ont rendu la méthode quantitative.

On prépare une solution du produit à étudier que l'on sature de sulfate de sodium ou d'ammonium pour diminuer la tension de vapeur de l'eau. Cette solution est placée en récipient hermétiquement bouché. Après quelques minutes, on prélève 5 à $10 \mathrm{ml}$ de gaz que l'on injecte dans le C.P.G.

Pour rendre la méthode quantitative, on ajoute au milieu un étalon interne qui entre dans l' " espace de tête » et qui donne un pic dont la surface sert de référence pour les autres. Cette méthode a été utilisée pour la bière $[34,69]$, le vinaigre [6], le café, le cacao, le thé [87].

\section{2) CONCENTRATION PAR LE FROID}

Ce type de technique a été décrit et utilisé en 1961 par Shapiro [92] et en 1963 par Bidmead [11]. La méthode consiste à éliminer la majeure partie de l'eau contenue dans la solution aqueuse traitée (bière, vin, produit laitier en suspension dans l'eau...) en la transformant en cristaux de glace.

La solution étudiée est plongée dans un mélange réfrigérant à $-10^{\circ} \mathrm{C}$, et est maintenue en agitation. Les cristaux de glace se forment au voisinage de la paroi du récipient ; à la fin du traitement il ne reste à l'état liquide qu'un petit cône central, dans lequel sont concentrés les composés aromatiques.

Moinas et al. [71] ont proposé, en 1973, une méthode analogue appliquée aux produits laitiers. Les constituants volatils sont ici isolés par entraînement sous pression réduite à l'aide d'argon selon une technique décrite par Moinas [70]. La solution obtenue est ensuite cryoconcentrée au bain-marie réfrigérant à $-10^{\circ} \mathrm{C}$. Cette opération est suivie d'une extraction à l'éther éthylique. L'analyse se fait ensuite par chromatographie en phase gazeuse suivie de spectrométrie de masse. Cette étude a porté sur des fromages du type Roquefort et Camembert.

\section{3) Distillation}

Les procédés de distillation ont été tout d'abord très utilisés pour l'étude des arômes de produits alimentaires. Cependant, il est maintenant reconnu que les traitements thermiques nécessaires dans ce type de techniques sont à l'origine de modifications des concentra- 
tions de certains composés ou encore de l'apparition d'artéfacts. Il conviendra donc de limiter ces traitements.

\section{a) Sous la pression atmosphérique}

Cette technique a été appliquée à la bière [35], aux jus de fruits [83, 94] aux fermentations alcooliques [7].

\section{b) Entraînement à la vapeur d'eau}

Prill et Hammer [84] ont utilisé cette technique pour la bière alors que Webb et Kepner [99] l'ont appliquée aux composés volatils du raisin. De même Honkanen et Karvonen [37] entraînent les substances volatiles des produits alimentaires par un courant de vapeur d'eau. Le distillat est récupéré dans des pièges à basse température et ensuite extrait par le chlorure d'éthyle.

Cette technique a été employée par Jackson [41] sur des bouillies aqueuses de fromage ou sur la phase grasse après séparation, par Patton [79]. Svensen et Ottestad [95] l'ont également utilisée pour des fromages.

\section{c) Distillation sous vide}

La distillation fractionnée sous vide permet d'obtenir des fractions de points d'ébullition différents, ce qui est intéressant pour l'analyse ultérieure. Cependant cette méthode présente deux inconvénients majeurs ; d'une part lorsque la taille des échantillons ne permet pas d'appliquer le procédé, d'autre part, lorsque les échantillons sont portés à des températures élevées pendant des périodes trop longues (ce qui peut entraîner des réarrangements moléculaires, des déshydratations, des cyclisations, des polymérisations, etc. qui modifient les distillats et faussent l'analyse).

Les premiers travaux ont porté sur la bière, le café, le thé...

C'est ainsi que Reymond et al. [87] évaporent des suspensions aqueuses de café, de cacao ou de thé à l'aide d'un évaporateur rotatif et recueillent le distillat dans des pièges réfrigérés. Le distillat étant ensuite extrait par le chlorure de méthylène. Strating et Venema [93], puis Ahrenst-Larsen et Levin-Hansen [2] appliquent la technique à la bière.

L'appareil de distillation moléculaire mis au point par Herz et Chang [36] et fonctionnant sous vide poussé $(0,01 \mathrm{~mm} \mathrm{Hg})$ permet de récupérer les composés volatils des produits alimentaires ou des boissons sans introduire les inconvénients dus aux températures élevées. Le distillat est recueilli dans des pièges maintenus à $-80^{\circ} \mathrm{C}$ et $-196^{\circ} \mathrm{C}$, puis extrait par un solvant adéquat.

Weurman [101] utilise un appareil d'évaporation sous vide sur un échangeur de température en verre pour concentrer l'acétone et l'acétate d'éthyle d'une solution aqueuse ; le distillat est condensé dans des pièges à $0^{\circ} \mathrm{C}$ et $-80^{\circ} \mathrm{C}$.

La technique de distillation sous vide a également été utilisée par Badings et al. [8] pour isoler les imines et par Bradley et Stine 
[15] qui ont ainsi comparé les produits volatils présents dans un Cheddar commercial et un Cheddar séché.

Forss et al. [30] ont décrit, en 1967, deux méthodes de concentration de composés volatils de point d'ébullition inférieur à $200^{\circ} \mathrm{C}$, à partir de solutions aqueuses diluées sans utilisation de solvants organiques. L'une des techniques est applicable à une gamme de volume très étendue $(5 \mathrm{ml}$ à $5000 \mathrm{ml})$ et comprend une distillation sous pression réduite ( 0 à 20 torrs) à travers un condenseur vertical maintenu à $0^{\circ} \mathrm{C}$. La deuxième méthode consiste à concentrer les composés à bas point d'ébullition et fait intervenir une sublimation sous vide poussé à travers un piège maintenu à $-50^{\circ} \mathrm{C}$. Cette deuxième technique peut être appliquée aux concentrés aromatiques obtenus par la première méthode; elle présente l'avantage de pouvoir s'appliquer à des composés que l'on ne peut récupérer par extraction à l'aide de solvants.

\section{4) ENTRAINEMENT DES COMPOSÉS VOLATILS PAR UN COURANT GAZEUX}

Cette méthode est employée en combinaison ou non avec le vide. L'entraînement se fait par barbottage ; les substances entraînées sont fixées sur du charbon actif ou dans des pièges réfrigérés (eau, glace : $0^{\circ} \mathrm{C}$, neige carbonique + acétone : $-80^{\circ} \mathrm{C}$, azote liquide : $-196^{\circ} \mathrm{C}$ ).

Deux types de systèmes sont employés :

- systèmes à balayage par un gaz à pression normale ;

- systèmes à balayage gazeux et sous vide.

a) Systèmes à balayage par un gaz et piégeage par le froid

En 1960, Nawar et Fagerson [74] ont réalisé un système de récupération et de dosage des substances aromatiques du vin, de certains fruits, etc.

Le gaz d'entraînement est l'azote. Après barbottage dans la solution étudiée et déshydratation dans une colonne de carbonate de potassium, la fraction aromatique est piégée dans un tube en acier inoxydable en forme de U plongé dans de l'azote liquide. Le gaz vecteur est ensuite recyclé grâce à une petite pompe (ce qui évite les pertes). Le piège est ensuite réchauffé et placé dans un circuit gazeux alimentant un C.P.G. Ce dispositif a été réalisé par Nawar et al. [76].

Swoboda [96] a utilisé ce système pour étudier l'arôme du lait, alors que Pollard et al. [82] l'ont adapté aux cidres.

Une technique analytique voisine a été utilisée en 1965 par Morgan et Day [73] pour des dosages de routine de produits à forte teneur en eau. L'appareillage utilisé se compose d'une fiole contenant l'échantillon et d'un système de fixation de l'arôme qui est porté à des températures pouvant atteindre $-196^{\circ} \mathrm{C}$. En cours de distillation, l'ensemble étanche est parcouru par un courant d'azote et les corps volatils sont piégés au niveau d'un serpentin en acier inoxydable 
immergé dans un mélange réfrigérant ou dans l'azote liquide. Dans une deuxième phase, le système de fixation est très rapidement et vigoureusement réchauffé et les gaz injectés directement dans un C.P.G. Les récupérations obtenues à partir d'échantillons aqueux d'acétaldéhyde et de butanone sont reproductibles à \pm 3 p. 100 près, alors que celles d'acétate d'éthyle et de diacétyle le sont à \pm 7 p. 100 près. L'entraînement à partir de lait réengraissé est plus difficile, ce qui oblige à doubler le temps de balayage pour obtenir des pics mesurables avec la même sensibilité. Grâce à ces nouvelles conditions, la reproductibilité des récupérations d'acétaldéhyde, de butanone et de diacétyle est de \pm 5 p. 100.

Mabbitt et Mc Kinnon [62] étudiant les substances volatiles du lait et de certains fromages, entraînent les fractions aromatiques dans un temps très court et les condensent directement dans la seringue d'injection dans le C.P.G.

La technique de Nawar et Fagerson [74] a été reprise par Hornstein et Crowe [39] ainsi que par Kepner et al. [48] en 1964. Cette dernière méthode permet la détermination quantitative de composants très volatils de solutions aqueuses sans isolement ou concentration préalables. Un étalon interne de référence est ajouté et la fraction la plus volatile est injectée dans le C.P.G. équipé d'un détecteur à ionisation de flamme. Ce procédé permet de réaliser rapidement des analyses de composés à des concentrations inférieures à 1 p.p.m.

b) Systèmes utilisant le piégeage par le froid après entraînement gazeux combiné avec le vide

Cette technique a été utilisée par Aurand et al. [6]. La distillation sous vide $(0,75 \mathrm{~mm} \mathrm{Hg})$ à $35^{\circ} \mathrm{C}$ dure $2 \mathrm{~h}$. Simultanément un barbottage d'azote entraîne l'arôme et le distillat est fixé dans plusieurs pièges réfrigérés, avant d'être étudié directement par C.P.G.

c) Systèmes basés sur l'adsorption sur solides après entraînement par un courant gazeux

Cette technique a été utilisée pour divers produits alimentaires et boissons mais elle n'a pas été adaptée au lait et aux produits laitiers. Moncrieff [72] en 1955 a montré que les substances volatiles des produits alimentaires peuvent être identifiées par leurs caractéristiques d'adsorption. L'entraînement se fait par un courant d'air et la condensation sur diverses substances : charbon activé, alumine activée, gel de silice... Cette technique a ensuite été reprise par d'autres chercheurs pour l'étude des fruits, du vin [78], de la bière, etc.

\section{5) MÉthodes D'EXtraction PaR solvants ORganiques}

Si le produit contient des lipides, on utilise des solvants qui dissolvent peu la matière grasse. En ce qui concerne les produits 
laitiers, il s'agit de l'acétonitrile utilisé par Wong et Parks en 1968 [105] pour l'analyse des fromages.

Le méthanol a été utilisé par Jackson [41] ainsi que l'isopentane.

\section{6) INJECTION DIRECTE}

Nous avons vu que dans les techniques de "Head-space " l'injection dans le C.P.G. peut être directe ; ce qui est le cas dans les travaux de Kroger et Patton [53] ou de Nawar et Fagerson [75].

Signalons à ce propos que l'on trouve dans la littérature de nombreux travaux basés sur l'analyse chromatographique en phase gazeuse avec détection par ionisation de flamme $[9,10,17,45,75,92$, 103, 104]. Cependant les détecteurs à ionisation de flamme sont en général moins sensibles que l'odorat; il faut donc toujours associer analyse et dégustation.

Depuis les travaux de James et Martin [42] en 1952, la chromatographie gaz liquide a été très utilisée et l'une des premières applications a été la chimie de l'arôme des aliments en 1956 par Dimick et Corse [21]. Cette technique est - comme nous le verrons plus loin - très employée car les échantillons sont de taille réduite et son haut pouvoir de séparation permet des analyses rapides.

\section{B. - Produits riches en matières grasses}

Les études relatives à ce type de produits peuvent être classées en deux groupes suivant que la matière grasse est extraite préalablement ou non. Celle-ci peut être séparée à froid $[3,89,90]$ par élution à l'aide de solvants convenables ou par centrifugation [88]. La phase grasse est alors soumise à une distillation sous vide poussé [29] ou bien analysée directement par chromatographie en phase gazeuse [60].

Certains composés tels que les lactones et les acides gras qui ont des points d'ébullition supérieurs à $200^{\circ} \mathrm{C}$, jouent un rôle important dans beaucoup de produits laitiers ; des techniques efficaces sont donc nécessaires pour les extraire.

En 1967, Angelini et al. [4] ont mis au point une méthode d'isolement et d'identification de tous les composés vaporisés au-dessus de $200^{\circ} \mathrm{C}$. L'opération est réalisée dans un appareil scellé en verre. l'isolement et le départ des composés se font au fur et à mesure de l'abaissement de la pression (jusqu'à $10^{-3}$ torr), les plus volatils se trouvant dans les fractions de tête.

Honkanen et Karvonen [38] ont utilisé une méthode de distillation sous vide avec entraînement des composés aromatiques par du gaz carbonique. Cette technique est limitée aux corps à haut point 
d'ébullition car le mélange initial est traité à l'aide de solvants organiques.

Forss et Holloway [29] ont comparé trois méthodes d'isolement de composés aromatiques à partir de l'huile de beurre à savoir :

- dégazage sous vide poussé ;

- distillation à l'aide d'azote dans des systèmes en " doigt de gant » et piégeage dans l'azote liquide ;

- distillation sous pression réduite à la vapeur d'eau.

Chacune de ces techniques présente des avantages et des inconvénients. Les rendements les plus élevés ont été obtenus avec la deuxième méthode. Les composés non aromatiques à point d'ébullition élevé interfèrent dans les analyses. La deuxième technique a ensuite été utilisée par Forss et al. [32] pour isoler les lactones à haut point d'ébullition et les acides gras.

De nombreux chercheurs ont utilisé les techniques de distillation sous vide $[8,15,16,37,56,58,59,63,77,81,88]$ alors que d'autres ont choisi des méthodes d'analyse directes.

Ce deuxième type de travaux consiste, soit en injections directes dans un chromatographe en phase gazeuse $[48,75]$ des composés présents dans l'atmosphère surmontant le produit à étudier, soit en injections après concentration en circuit fermé ou entraînement par un courant d'azote [3, 41, 55, 73].

En 1972, Arnold et Barnhart [5] ont employé une technique consistant à mélanger la phase grasse au support de chromatographie. Le mélange à 30 p. 100 de lipides est placé dans une colonne en acier inoxydable et balayé par un courant d'azote qui véhicule après chauffage les fractions aromatiques, séparées de la matière grasse, à un chromatographe branché en série avec le dispositif de séparation. Les résultats montrent que le taux de récupération est plus élevé qu'après isolement préalable de la matière grasse.

Certaines techniques d'extraction de l'arôme font appel à l'action de substances choisies en fonction du fait qu'elles ne dissolvent pas la matière grasse. C'est ainsi que certains chercheurs ont employé l'acétonitrile [105] dans une colonne remplie d'un mélange de l'échantillon de fromage à analyser et de célite. Les résultats ont montré que la première fraction éluée est suffisamment concentrée pour permettre la chromatographie en phase gazeuse sans évaporation du solvant. D'autres études ont été réalisées à l'aide de méthanol et d'isopentane $[41,60]$.

Les acides gras sont en général dosés par chromatographie en phase gazeuse $[33,40,46,54,66]$.

Citons enfin les techniques utilisant la distillation à la vapeur d'eau. L'échantillon étudié est réduit en une pâte de laquelle on extrait ou non la matière grasse $[79,84,95,98]$. 


\section{C. - Application à certains produits laitiers}

\section{1) LAIT}

Bien que la sensation dominante lors de l'absorption de lait soit certainement due à sa structure colloïdale, il semble que l'odeur caractéristique du lait frais soit due à des corps de faible poids moléculaire : acétone, acétaldéhyde, sulfure de méthyle, traces d'acides gras de $\mathrm{C} 4$ à $\mathrm{C} 10$ associés à la membrane des globules gras : Kintner et Day [51], $\Delta$-lactones: Kinsela et al [50] ont montré, en 1967, que de simples traces de $\Delta$-lactones, composés à goût de noix de coco, sont aisément détectées dans le lait alors que 5 à 10 p.p.m. sont souhaitables dans le beurre.

Depuis les travaux de Patton et al. [80] de 1956 signalant la présence de sulfure de méthyle dans le lait, plusieurs études ont été réalisées mettant en évidence son importance [27, 47].

Les levains - et en particulier ceux de beurrerie - ont été étudiés par de nombreux chercheurs qui se sont appliqués à définir les conditions optimales de production de composés aromatiques.

Dans les travaux de Lindsay et al. [61] publiés en 1965, la source de composés volatils est une culture de souches lactiques mixtes dont Str. diacetilactis. Le milieu de culture a été préparé en incorporant 0,2 p. 100 de citrate de sodium à du lait cru entier et en chauffant au bain-marie bouillant pendant $1 \mathrm{~h}$. On inocule à $1 \mathrm{p}$. 100 et on incube à $21^{\circ} \mathrm{C}$. Pour parvenir à l'optimum de flaveur, l'acidité des cultures mères est maintenue entre 0,68 p. 100 et 0,75 p. 100. La culture - ainsi que le lait de contrôle - est distillée à basse température sous pression réduite. Le mélange de composés aromatiques est isolé à partir de distillats aqueux par extraction à l'éther éthylique pur. Après élimination de l'excès d'éther les «concentrés aromatiques " sont analysés par chromatographie en phase gazeuse et spectrométrie de masse. La corrélation des deux techniques a permis l'identification de plusieurs composants neutres : aldéhydes, cétones, alcools, esters.

\section{2) Fromages}

Jusqu'à ces dernières années, la plupart des études d'arômes fromagers ont été réalisées aux Etats-Unis, ce qui explique l'abondance de travaux sur le Cheddar $[19,20,31,64,68]$.

La base de l'arôme du Cheddar est due aux levains comme l'ont montré Reiter et al. [85] en étudiant l'influence des micro-organismes et des enzymes. Les composés principaux intervenant sont l'acide acétique ainsi que les acides butyrique et caproïque [31] et les composés neutres volatils, bien que certains composés soufrés comme l'hydrogène sulfuré, les méthyl-mercaptans et le sulfure de méthyle jouent également un rôle non négligeable. Les composés neutres volatils du Cheddar ont été étudiés par Mc Gugan et al. [64] en 1968, en utilisant la technique mise au point par Mc Gugan et Howsam 
[63]. Cette technique, déjà mentionnée plus haut, consiste en une distillation sous vide à partir de matière grasse obtenue par centrifugation à grande vitesse. Les vapeurs des échantillons sont condensées dans une série de pièges, la chromatographie est effectuée dans des colonnes capillaires. Les temps de rétention relatifs des substances volatiles sont comparés à ceux de composés connus sur des chromatogrammes obtenus à partir de conditions différentes. Des colonnes capillaires plus longues peuvent ultérieurement augmenter la résolution, mais la faible sensibilité relative du détecteur à ionisation de flamme aux composés soufrés constitue un handicap.

Trois types de fromages ont ainsi été analysés. Deux ont été fabriqués aseptiquement, sans levains, le troisième a été fait avec des levains en cuve ouverte. La chromatographie en phase gazeuse et la spectrométrie de masse ont permis de détecter les mêmes composés dans le fromage sans levains - qui s'est révélé très peu aromatique - que dans celui réalisé avec des levains et dont l'arôme est typique.

Plus récemment, en 1973, Manning et Heather [68] ont distillé du Cheddar et l'ont concentré à $-80^{\circ} \mathrm{C}$. Les chromatogrammes du distillat ont été obtenus en échantillonnant la vapeur au-dessus du concentré en utilisant une colonne tubulaire ouverte d'Apiezon de $15 \mathrm{~m}$ et une colonne en verre de $1,5 \mathrm{~m}$ remplie de Porapak Q. Les composés responsables de l'arôme détectés dans le gaz effluent sont identifiés en couplant le chromatographe à un spectromètre de masse. Parmi les composés identifiés, $\mathrm{H}_{2} \mathrm{~S}$, le sulfure de méthyle et le diacétyle ont les arômes les plus intenses.

Depuis quelques années d'autres types de fromages ont été étudiés en utilisant des techniques analogues à celles que nous venons de décrire.

C'est ainsi que Adda et Dumont [1] ont analysé les fromages à pâte molle, Dumont et al. [24, 25, 26] le Parmesan, les pâtes molles lavées, le vacherin...

Outre ces travaux relatifs à la fraction aromatique que nous qualifierons d'utile, on trouve dans la littérature beaucoup d'études concernant les défauts de flaveur $[8,13,18,52,57,67,79,90,97]$.

De nombreux chercheurs, nous venons de le voir, ont choisi d'analyser la phase lipidique en supposant qu'elle représente entièrement l'arôme du produit étudié. Dumont et Adda [23] ont montré, en 1972, que la distillation sous vide poussé de la matière grasse peut être efficace si l'on ne prétend pas réaliser une étude globale de l'arôme.

Ces chercheurs ont comparé en analyse chromatographique le fromage à la matière grasse isolée en utilisant une technique de distillation sous vide poussé et la méthode plus simple de distillation sous reflux décrite par Forss et Holloway [29]. Outre la constatation 
que nous avons déjà énoncée plus haut, cette étude montre que la distillation sous reflux peut être utilisée efficacement pour analyser les fractions aromatiques de tête même en travaux de routine.

(à suivre)

Reçu pour publication en juin 1975.

\section{Bibliographie}

[1] Adda (J.) et Dumont (J. P.) (1974). - Le Lait, 54 (531-532), 1-21.

[2] Ahrenst-Larsen (B.) and Levin-Hansen (H.) (1964). - Wallerstein Lab. Comm., 92, 41-49.

[3] Anderson (D. F.) and Day (E. A.) (1965). - J. Dairy Sci., 48 (2), 248-249.

[4] Angelini (P.), Forss (D. A.), Bazinet (M. L.) and Merritt Jr. (C.) (1967). J. Amer. Oil Chemists' Soc., 44, 26.

[5] ARnold (R. G.) and Barnhart (H. M.) (1972). - J. Dairy Sci., 55 (8), 10691072.

[6] Aurand (L. W.), Singleton (J. A.), Bell (T. A.) and Etchells (J.L.). J. Food Sci., 1966, 31 (2), 172-177 (a) et 1965, 30 (2), 288-295 (b).

[7] AyrapaA (T.) (1967). - J. Inst. Brew., 73, 17.

[8] Badings (H. T.), Stadhouders (J.) and Van Duin (H.) (1968). - J. Dairy Sci., 51 (1), 31.

[9] Bassette (R.) and Claydon (T. J.) (1956). - J. Dairy Sci., 48, 775.

[10] Bassette (R.), Ozeris (S.) and Whitnah (C. H.) (1962). - Anal. Chem., 34, $1540-1543$.

[11] Bidmead (D. S.) (1963). - Rec. Adv. in Food. Sci., 3, 158-164, ed. Muil Leitch J., London Butterworth.

[12] Bills (D. D.), Khatri (L. L.) and Day (E. A.) (1963). - J. Dairy Sci., 46, (12), 1342.

[13] Bills (D. D.), Morgan (M. E.), Libbey (L. M.) and Day (E. A.) (1965). J. Dairy Sci., 48, 1168-1173.

[14] Bills (D. D.), Willits (R. E.) and DaY (E. A.) (1966). - J. Dairy Sci., $49,681$.

[15] Bradley (R. L.) Jr. and Stine (C. M.) (1968). - J. of Gas Chromat, 6, 344.

[16] Bruyn (J. De) and Schogt (J. C. M.) (1961). - J. Am. Oil Chemist' Soc., 38, 40.

[17] Buttery (R. G.) and Teranishi (R.) (1961). - Anal. Chem., 33, 1439-1441.

[18] Czulak (J.) (1959). - Australian J. Dairy Tech., 14, 177.

[19] Day (E. A.), Bassette (R.) and Keeney (M.) (1960). - J. Dairy Sci., 43, 463.

[20] DAY (E. A.) and LibBEy (L. M.) (1964). - J. Food Sci., 29, 583-589.

[21] Dimick (K. P.) and CoRse (J.) (1956). - Food Technol., 10, 360-364.

[22] Dumont (J. P.) et Adda (J.) (1973). - Le Lait, 53, 12-22.

[23] Dumont (J. P.) et Adda (J.) (1972). - Le Lait, 52, 311-323.

[24] Dumont (J. P.), Roger (S.) et Adda (J.) (1974). - Le Lait, 54, 31-43.

[25] Dumont (J. P.), Roger (S.) et AdDA (J.) (1974). - Le Lait, 54, 386-396.

[26] Dumont (J. P.), Roger (S.), Cerf (P.) et AddA (J.) (1974). - Le Lait, 54, 243-251.

[27] Dunham (J. R.), Ward (G.), Bassette (R.) and Reddy (M. C.) (1968). J. Dairy Sci., 51, 199. 
[28] Fonss (D. A.) (1969). - Food Prod. Develop., 3 (4), 76.

[29] Forss (D. A.) and Holloway (G. L.) (1967), - J. Am. Oil Chem. Soc., 44 (10), 572.

[30] Forss (D. A.), Jacobsen (V. M.) and Ramshaw (E. H.) (1967). - J. Agr. Food Chem., 15 (6), 1104-1107.

[31] Forss (D. A.) and Patton (S.) (1966). - J. Dairy Sci., 49, 89-91.

[32] Forss (D. A.), Stark (W.) and Urbach (G.) (1966), - J. Dairy Res,, 34, 131.

[33] Francesco (F. De) Avancini (D.), Maglitto (C.) e Gandini (C.) (1961), - Riv. ital. Sostanze grasse., 38, 307.

[34] Harrison (G. A. F.), Byrne (W. J.) and Collins (E.) (1965). - J. Inst. Brew., $71,336$.

[35] Hashimoto (N.) and Kuroiwa (Y.) (1966). - J. Inst. Brew., 72, 151.

[36] Herz (K. O.) and Chang (S. S.) (1966). - J. Food Sci., 31, 937-940.

[37] Honkanen (E.) and Karvonen (P.) (1963). - Act. Chem. Scand., 17, 13571362.

[38] Honkanen (E.), Karvonen (P.) and Virtanen (A. I.) (1964). - Act. Chem. Scand., 18, 612.

[39] Hornstein (I.) and Crowe (P. F.) (1962). - Anal. Chem., 34, 1354-1356.

[40] Iyer (M.), Richardson (T.), Amundson (C. M.) and Boudreau (A.) (1967). J. Dairy Sci., 50, 285.

[41] JaCkSON (H. W) (1958). - Perfumery and essential oil record, 49, 256.

[42] JAMES (A. T.) and MARTin (A. J. P.) (1952), - Analyst., 77, 915.

[43] Jennings (W. G.) (1957). - J. Dairy Sci., 40, 271-279.

[44] Jennings (W. G.) and Nursten (H. E.) (1967). - Anal. Chem., 39, 521-523.

[45] Jennings (W. G.), Viljhalmsson (S.) and Dunkley (W. L.) (1962). - J. Food Sci., 27, 306-308.

[46] Кавот (F. J.), Averill (W.) and Ettre (L. S.) (1964). - Riv. ital. Sostanze grasse., 41, 121.

[47] Keenan (T. W.), and Lindsay (R. C.) (1968). - J. Dairy Sci., 51, 112.

[48] Kepner (R. E.), Maarse (H.) and Strating (J.) (1964). - Anal. Chem., $36,77$.

[49] Kepner (R. E.), Strating (J.) and Weurman (C.) (1963). - J. Inst. Brew., $69,399$.

[50] Kinsella (J. E.), Patton (S.) and Dimick (P. S.) (1967). - J. Amer. Oil Chemists' Soc., 44, 449.

[51] Kintner (J. A.) and Day (E. A.) (1965). - J. Dairy Sci., 48, 1575.

[52] Kristoffersen (T.), Gould (I. A.) and Purvis (G. A.) (1964). - J. Dairy Sci., 47, 599-603.

[53] Kroger (M.) and Patton (S.) (1964). - J. Dairy Sci., 47, 296.

[54] Kuzdzal-Savoie (S.) et Kuzdzal (W.) (1967). - Le Lait, 47, 487-494.

[55] Langler (J. E.) (1966). - Diss. Abs., sect B, 27 (8), 2625.

[56] Langler (J. E.), Libbey (L. M.) and Day (E. A.) (1967). - J. Agr. Food Chem., 15 (3), 386-391.

[57] Lawrence (R. C.) (1963). - J. Dairy Res., 30, 235.

[58] Lea (C. H.) and Swoboda (P. A. T.) (1962). - J. Sci. Food Agr., 13, 148.

[59] LibBey (L. M.), Bills (D. D.) and DAY (E. A.) (1963). - J. Food Sci., 28 (3), 329-333.

[60] Liebich (H. M.), Douglas (D. R.), Bayer (E.) and Zlatkis (A.) (1970). J. of Chrom. Sc., 8, 351-355.

[61] Lindsay (R. C.), Day (E. A.) and Sandine (W. E.) (1965). - J. Dairy Sci., 48, 1566-1574. 
[62] Mabbitt (L. A.) and Mc Kinnon (G.) (1963). - J. Dairy Res., 30, 359.

[63] Mc Gugan (W. A.) and Howsam (S. G.) (1962). - J. Dairy Sci., 45, 495-499.

[64] Mc Gugan (W. A.), Howsam (S. G.), Elliott (J. A.), Emmons (D. B.), Reiter (B.) and SHARPe (E.) (1968). - J. Dairy Res., 35, 237.

[65] Mackay (D. A. M.), Lang (D. A.) and Berdick (M.) (1961). - Anal. Chem., $33,369$.

[66] Man (J. M. DE) (1964). - J. Dairy Sci., 47, 546.

[67] Manning (D. J.) (1974). - J. Dairy Res., 41, 81-87.

[68] Manning (D. J.) and Robinson Heather (M.) (1973). - J. Dairy Res., 40 (1), 63-75.

[69] MaUle (D. R.) (1967), - J. Inst. Brew., 73, 351-361.

[70] Moinas (M.) (1973). - Mitt. Lebensmitteluntersuch. u. Hug., 64 (1), 60.

[71] Mornas (M.), Groux (M.) et Horman (I.) (1973). - Le Lait, 53, 601-609.

[72] MoncriefF (R. W.) (1955). - Foods, 24, 154-157.

[73] Morgan (M. E.) and Day (E. A.) (1965). - J. Dairy Sci., 48, 1382-1384.

[74] NaWAR (W. W.) and Fagerson (I. S.) (1960). - Anal. Chem., 32 (11), 1534.

[75] NAWAR (W. W.) and Fagerson (I. S.) (1962). - Food Technol., 107-109.

[76] Nawar (W. W.), Sawyer (F. M.), Beltran (E. G.) and Fagerson (I. S.) (1960). - Anal. Chem., 32, 1534.

[77] O'Keefe (P. W.), Libbey (L. M.) and Lindsay (R. C.) (1969). - J. Dairy Sci., $52,888$.

[78] Ough (C. S.) and Amerine (M. A.) (1959). - Amer. J. Enol., 10 (1), 17-19.

[79] Patton (S.) (1950). - J. Dairy Sci., 33, 680.

[80] Patton (S.), Forss (D. A.) and Day (E. A.) (1956). - J. Dairy Sci., 39, 1469.

[81] Patton (S.) and Tharp (B. W.) (1959). - J. Dairy Sci., 42, 49-55.

[82] Pollard (A.), Kieser (M. E.), Stevens (P. M.) and Tucknott (O. G.) (1965). J. Sci. Food Agric., 16, 384-389.

[83] Pribela (A.) (1965). - Biologia Esckoel, 3, 173.

[84] Prill (E. A.) and Hammer (B. W.) (1939). - J. Dairy Sci., 22, 67.

[85] Reiter (B.), Fryer (T. F.), Pickering (A.), Chapman (H. R.), Lawrence (R. C.) and SHARPE (M. E.) (1967). - J. Dairy Res., 34, 257.

[86] Reiter (B.), Fryer (T. F.), Sharpe (M. E.) and Lawrence (R. C.) (1966). J. Appl. Bacteriol., 29, 281.

[87] Reymond (D.), Mueggler-Chavan (F.), Viani (R.), Vuataz (L.) and Egli (H.) (1966). - J. Gas Chrom., 28.

[88] Scarpellino (R.), and Kosikowski (F. V.) (1962). - J. Dairy Sci., 45, 495.

[89] Schwartz (D. P.) and Parks (O. W.) (1963). - J. Dairy Sci., 46 (9), 989-990.

[90] Schwartz (D. P.) and Parks (O. W.) (1963). - J. Dairy Sci., 46 (10), 1136.

[91] Shapiro (J.) (1961). - Science, 133, 2063.

[92] Singleton (V. L.) (1961). - An. J. of Enology, 12 (1), 1-8.

[93] Strating (J.) and Venema (A.) (1961). - J. Inst. Brew., 525.

[94] Sundt (E.) and Winter (M.) (1962), - Helv. Chim. Acta, 45, 2212.

[95] Svensen (A.) and OTtestad (E.) (1969). - Meieriposten., 58 (3), 50,57 et (4), 77-81.

[96] Swoboda (P. A. T.) (1962). - 4 th., Symp. on gaz Chrom., 29.

[97] Walker (J. R. L.) (1961). - J. Dairy Res., 28, 1.

[98] WALSh (B.), and Cogan (T. M.) (1973). - Appl. Microbiol., 26 (5), 820-825.

[99] WebB (A. D.) and KePNER (R. E.) (1957). - Food. Res., 22, 384-395.

[100] Weurman (C.) (1961). - Food Technol., 15, 531.

[101] Weurman (C.) (1961). - J. Food. Sci., 26, 670-672.

[102] Weurman (C.) (1963). - Café, cacao, thé., 7 (4), 341-346.

[103] Weurman (C.) (1969). - J. Agr. Food Chem., 17 (2), 370-384.

[104] Weurman (C.), Groenen (P. J) and Van Gemert (L. J.) (1970). - Die Nahrung., $14(7), 607$.

[105] Wong (N. P.) and Parks (O. W.) (1968). - J. Dairy Sci, 51, 1768. 